\title{
Is It Necessary to Measure T4 In Screening of Congenital Hypothyroidism in Preterm Infants?
}

\author{
Mitra Radfar ${ }^{1}$, Minoo Fallahi' ${ }^{2}$, Naeeme Taslimi Taleghani ${ }^{2}$, Maryam Kazemi Aghdam ${ }^{3}$, Sara Khoshkhu ${ }^{2}$ and \\ Salehe Tajalli ${ }^{2}$ \\ ${ }^{1}$ ShahidBeheshti University of Medical Sciences, Imam Hussein Hospital, Iran \\ ${ }^{2}$ Neonatal Health Research Center, Shahid Beheshti University of Medical Sciences, Iran \\ ${ }^{3}$ Pediatric Pathology Research Center, Shahid Beheshti University of Medical Sciences, Iran \\ Submission: September 02, 2018; Published: November 30, 2018 \\ *Corresponding author: Minoo Fallahi, ShahidBeheshti University of Medical Sciences, Imam Hussein Hospital, Iran
}

Abstract

Introduction: Immaturity of hypothalamus-pituitary-thyroid axis in preterm infants causes the low level of thyroxin hormone and hypothyroxinemia of prematurity (HOP). In our country, Iran TSH that is measured by filter paper is the only laboratory test for screening of hypothyroidism and the missing of the HOP is concerning. This research was done to evaluate the rate of HOP.

Material and Methods: This prospective-descriptive study was conducted in 2017 in our hospital. In the participants included preterm infants with gestational age $\leq 34$ weeks or birth weights $\leq 2000$ grams, measurement of TSH and T4 were done by blood sampling instead of filter paper and the values of thyroid function tests were analyzed.

Results: In total 475 preterm newborns in the first sampling and 203 cases in the second one were enrolled, 236(49.7\%) neonates were male. Mean gestational age and birth weight were 31.46 weeks and 1522.59 grams respectively. In overall, in the first blood sampling, 100 of $475(21 \%)$ preterm infants had low T4 ( $13.1 \%$ related to HOP and $8.3 \%$ belonged to hypothyroidism) , but in second sampling, in the 50 from 203 cases $(24.7 \%$ ), the low levels of T4 were observed ( $14.2 \%$ related to HOP and $10.3 \%$ belonged to hypothyroidism).In comparisons the values of T4 in both of sampling: in 24 cases (11.8\%) persistent hypothyroxinemia ,26(12.8\%) delayed hypothyroxinemia and in $29(14.2 \%)$ transient forms of hypothyroxinemia were detected.

Conclusion: Our research resulted that the rate of HOP is remarkable and measurement of T4 in the screening of preterm infants is recommended.

Keywords: Preterm infants; Hypothyroxinemia; Congenital hypothyroidism

\section{Introduction}

Congenital hypothyroidism is the most preventable causes of mental retardation in childhood [1] provided that the diagnosis and treatment are given on time. Universal neonatal screening of hypothyroidism started in 1972 in Canada [2] Some countries measure TSH as the first laboratory exam for the screening of this disease and some other countries use T4 as the first screening test. Screening tools of hypothyroidism in term and preterm infants is similar and dried blood spot on filter paper is the usual laboratory methods for checking of TSH or T4 as the mass screening in many countries [3], although the frequency of screening in preterm infants is more than term babies (four times versus once)[4] .In Iran, the mass screening of hypothyroidism in neonates was started from 2005 [5] and resemble other countries, TSH by the dried blood spot on filter paper, is the first laboratory test for screening. In cases with TSH more than 5mg/ dl in filter paper blood spot, by recall the parents of patients, T4 and TSH are evaluated at a reference laboratory, then as a standard protocol, measurement of T4 is provided that the high level of TSH. Immaturity of hypothalamus-pituitary-thyroid axis causes the low level of thyroxin hormone in preterm infants that spontaneously rises to normal levels by 6-8-weeks of life [6],in fact hypothyroxinemia of prematurity(HOP) that is defined by the low level of T4 and free T4, concurrent with a normal level of TSH observed in $15-20 \%$ of preterm infants [7] . Considering that thyroid hormones are critical for the normal development of the brain, the low significant levels of $\mathrm{T} 4 \mathrm{can}$ be dangerous for developing brain of preterm infants. Some previous researches [8] showed that the low levels of T4 can cause adverse effects 
in preterm neonates such as intraventricular hemorrhage(IVH), neurodevelopmental delay, deficits in cognitive, motor, languages, attention and memory [9], particularly severe hypothyroxinemia which could be associated with a poor outcome in premature babies.

Thyroid hormone level can be a predictor of neonatal outcome for morbidities such as infection, hypoxia, patent ductus arteriousus (PDA), necrotizing enterocolitis (NEC) [10]. Considering that, in most of NICUs in Iran, as protocols of the health ministry, screening of hypothyroidism is done just with measurement of TSH by dried blood spot on filter paper, there is a risk of missed severe hypothyroxinemia and its possible consequences in this high-risk infant. This research was done to evaluate the rate of low thyroid hormone in preterm babies admitted to our NICU for the detection of the rate of HOP in these high-risk infants. In severe decreased levels of thyroxin, a closer follow up test for the evaluation of changes in this hormone was recommended. If our research showed the high incidence of HOP, measurement of $\mathrm{T} 4$ beside to TSH will suggest in the screening program of preterm babies.

\section{Material and Methods}

This prospective-descriptive study was conducted in 2017 in Mofid Children's Hospital and Imam Hossein Hospital affiliated by Shahid Beheshti University of Medical Sciences in Tehran/ Iran. Participants included preterm infants with gestational age $\leq 34$ weeks or birth weight $\leq 2000$ grams were admitted in the NICU. Based on the policy of thyroid screening in our country Iran, the first screening of congenital hypothyroidism was done on the third to fifth days of birth just by TSH measurement with a dried blood spot on filter paper method and in preterm babies repeated screening was done in $14^{\text {th }}, 42^{\text {th }}$ and $70^{\text {th }}$ days of life. In eligible patients in our research, measurement of TSH and T4 were done by blood sampling in laboratory part of hospitals by enzyme-linked immunosorbent assay (ELISA) method and the use of filter paper was ignored. Due to the discharge of some neonates from the hospital, we had the results of first screening in all patients (476 cases) and the second sampling was done in 203 patients who stay in the hospital at the time of second sampling. Unfortunately, we didn't access to the results of the third (on the $42^{\text {nd }}$ days of life) and the fourth (on the $70^{\text {th }}$ days of life) checking of thyroid function test which was done after discharge of hospital. The ethical committee of Shahid Beheshti University of Medical Sciences approved our research and written consent was taken from the parents of patients. Exclusion criteria's were the newborns treated by levothyroxine before the starting of our research, and those screened with filter paper. The timing of blood sampling was at least 3-5 days of life or later at the first day of admission if the screening was not done before.

Hypothyroxinemia defines as the low level of T4 concurrent with a normal level of TSH and hypothyroidism defined as the low level of T4 along with a high level of TSH. Regard to the detection of severe hypothyroxinemia, we analyzed the results of
T4 in 3 divided subgroups and defined the normal levels of T4: $\geq 7 \mathrm{ng} / \mathrm{dl}$, mild hypothyroxinemia as the values between $5-7 \mathrm{ng} / \mathrm{dl}$, moderate one as the level of T4 between $3-5 \mathrm{mg} / \mathrm{dl}$ and $\leq 3 \mathrm{mg} / \mathrm{dl}$ defined as the severe forms of disorder. The normal level of TSH was considered $\leq 5 \mathrm{mg} / \mathrm{dl}$ and the values between $5-10 \mathrm{mg} / \mathrm{dl}, 10$ $20 \mathrm{mg} / \mathrm{dl}$ and $\geq 20 \mathrm{mg} / \mathrm{dl}$ concurrent with low level of T4, defined as mild, moderate and severe forms of hypothyroidism. In cases with repeated thyroid tests with no treatment by levothyroxine, their results were analyzed too. Unfortunately, given that we didn't access to the specific laboratory kit of free T4, we ignore that, and the analysis of our study was done just the basis of TSH and T4 values. For detection of INH brain sonography was done by pediatric sinologist and based on the extension of bleeding, IVH was expressed to 1-4 grade. Grade 1defined to germinal matrix hemorrhage, grade 2 defined to intraventricular hemorrhage without ventriculomegaly, grade 3 means intraventricular hemorrhage with ventriculomegaly and grade 4 intraparenchymal hemorrhage. The variables such as gestational age, birth weight, sex, and the time of laboratory test (based on the chronological age of newborn) and the values of T4 and TSH were analyzed. Data analysis was performed in SPSS version 20 (SPSS Inc., Chicago, IL) and the quantitative variables were expressed in mean and standard deviation (SD). In addition, repeated measures ANOVA were used to evaluate the differences between the variables. P-value of less than 0.05 was considered statistically significant.

\section{Results}

Table 1: Demographic data of patients and frequency of abnormal thyroid function tests.

\begin{tabular}{|c|c|}
\hline Patients characteristics & N (\%) \\
\hline \multicolumn{2}{|c|}{ Gestational age(weeks) } \\
\hline $23-25$ & $6(1.3 \%)$ \\
\hline $25-28$ & $65(13.7 \%)$ \\
\hline $28-32$ & $217(45.7 \%)$ \\
\hline $32-34$ & $109(22.9 \%)$ \\
\hline$>34$ & $78(16.4 \%)$ \\
\hline \multicolumn{2}{|c|}{ Birth weight(grams) } \\
\hline$<750$ & $9(1.9 \%)$ \\
\hline $751-1000$ & $30(6.3 \%)$ \\
\hline $1001-1250$ & $66(13.9 \%)$ \\
\hline $1251-1500$ & $89(18.7 \%)$ \\
\hline $1501-2000$ & $262(55.2 \%)$ \\
\hline$>2000$ & $19(4 \%)$ \\
\hline \multicolumn{2}{|c|}{ First $T 4(n g / m l)(n=463)$} \\
\hline$<3$ & $22(4.6 \%)$ \\
\hline 5-Mar & $34(7.2 \%)$ \\
\hline 7-May & $44(9.3 \%)$ \\
\hline$>7$ & $375(78.9 \%)$ \\
\hline \multicolumn{2}{|c|}{ First TSH(ng/ml) $(\mathrm{n}=463)$} \\
\hline$\leq 5$ & $299(62.9 \%)$ \\
\hline 10-May & $116(24.4 \%)$ \\
\hline
\end{tabular}




\section{Academic Journal of Pediatrics \& Neonatology}

\begin{tabular}{|c|c|}
\hline 20-Oct & $29(6.1 \%)$ \\
\hline$>20$ & $19(4 \%)$ \\
\hline \multicolumn{2}{|c|}{ Second T4(ng/ml) $(\mathrm{n}=203)$} \\
\hline$<3$ & $11(5.4 \%)$ \\
\hline 5-Mar & $12(5.9 \%)$ \\
\hline 7-May & $27(13.3 \%)$ \\
\hline$>7$ & $153(75.3 \%)$ \\
\hline \multicolumn{2}{|c|}{ Second TSH $(\mathrm{ng} / \mathrm{ml})(\mathrm{n}=203)$} \\
\hline$\leq 5$ & $119(58.6 \%)$ \\
\hline 10-May & $56(27.5 \%)$ \\
\hline 20-Oct & $20(9.8 \%)$ \\
\hline$>20$ & $8(3.9 \%)$ \\
\hline
\end{tabular}

In total 475 preterm newborns were enrolled in the study, $236(49.7 \%)$ neonates were male and $239(50.3 \%)$ were female. Mean gestational age and birth weight of the newborns were 31.46weeks (23-40weeks) and 1522.59grams (600-2000grams) respectively. From total patients, 203 cases were stayed in our NICU on the age of second thyroid screening test thus enrolled in the second sampling of thyroid function tests. None of our cases had the repeated screening test of third and fourth in the hospital. The most frequent gestational age of our patients was 28-32 weeks in 217 (45.7\%) of cases, and 32-34 weeks in 109(22.9\%) of patients (Table 1), the most frequent birth weight of our patients was 1501-2000 grams in 262 (55.2\%) cases (Table 1).

Mean age of the first sampling in neonates was 9.36 days (3-60 days) with SD:10.14 days and for the second sampling was 27.8 days (4-72 days) with SD:14 days. Demographic data of patients and the results of the first and second tests were shown in (Table 1).

In the first sampling in $375(78.9 \%)$ and $299(62.9 \%)$ had the normal range of T4 and TSH respectively. In second sampling in the $153(75.3 \%)$ and $119(58.6 \%)$ had the normal values of $\mathrm{T} 4$ and TSH respectively.

(Table $2 \& 3$ ) showed the correlations between the levels of first and second sampling of T4 and demographic characteristics of patients. As we shown, in the first sampling of newborns, there was the statistically significant difference between the level of T4 and birth weight $(\mathrm{p}=<0.01)$ of patients, without any difference between sex and gestational age and the T4 level of patients ( $p=0.055$ and $p=0.084$ respectively). In second sampling the differences between sex $(p=<0.018)$, and birth weight $(p=0.36)$ of patients and the T4 levels were statistically significant without any significant difference with gestational age $(p=0.06)$, means that hypothyroxinemia was male predominant and was most frequent in 1500-2000 grams of birth weight.

Table 2: Correlations between the levels of first sampling T4 and demographic characteristics of patients.

\begin{tabular}{|c|c|c|c|c|c|c|}
\hline & $<3 \mathrm{~N}(\%)$ & 5-Mar N (\%) & 7-May N (\%) & $>7 \mathrm{~N}(\%)$ & Total N (\%) & P-value \\
\hline \multicolumn{7}{|l|}{ Sex } \\
\hline Male & $11(2.3 \%)$ & $19(4 \%)$ & $28(5.8 \%)$ & $178(37.4 \%)$ & $236(49.6 \%)$ & \multirow{3}{*}{0.055} \\
\hline Female & $11(2.3 \%)$ & $15(3.1 \%)$ & $16(3.3 \%)$ & $197(41.4 \%)$ & $239(50.3 \%)$ & \\
\hline Total & $22(4.6 \%)$ & $34(7.1 \%)$ & $44(9.2 \%)$ & $375(78.9 \%)$ & $475(100 \%)$ & \\
\hline \multicolumn{7}{|l|}{ G.A (weeks) } \\
\hline $23-25$ & $1(0.2 \%)$ & $2(0.4 \%)$ & $1(0 \%)$ & $2(0.4 \%)$ & $6(1.2 \%)$ & \multirow{6}{*}{0.084} \\
\hline $25-28$ & $5(1.0 \%)$ & $7(1.4 \%)$ & $9(1.8 \%)$ & $44(9.2 \%)$ & $65(13.6 \%)$ & \\
\hline $28-32$ & $9(1.8 \%)$ & $14(2.9 \%)$ & $18(3.7 \%)$ & $176(37.0 \%)$ & $217(45.6 \%)$ & \\
\hline $32-34$ & $3(0.6 \%)$ & $7(1.4 \%)$ & $11(23 \%)$ & $88(18.5 \%)$ & $109(22.9 \%)$ & \\
\hline$>34$ & $4(0.8 \%)$ & $4(0.8 \%)$ & $5(1.0 \%)$ & $65(13.6 \%)$ & $78(16.4 \%)$ & \\
\hline Total & $22(4.6 \%)$ & $34(7.1 \%)$ & $44(9.2 \%)$ & $375(78.9 \%)$ & $475(100 \%)$ & \\
\hline \multicolumn{7}{|c|}{ Birth weight(grams) } \\
\hline$<750$ & $1(0.2 \%)$ & $1(0.2 \%)$ & $2(0.4 \%)$ & $5(1.0 \%)$ & $9(1.8 \%)$ & \multirow{7}{*}{$<0.001$} \\
\hline $751-1000$ & $3(0.6 \%)$ & $6(1.2 \%)$ & $3(0.6 \%)$ & $18(3.7 \%)$ & $30(6.3 \%)$ & \\
\hline $1001-1250$ & $2(0.4 \%)$ & $8(1.6 \%)$ & $7(1.4 \%)$ & $49(10.3 \%)$ & $66(13.8 \%)$ & \\
\hline $1251-1500$ & $5(1.0 \%)$ & $6(1.2 \%)$ & $9(1.8 \%)$ & $69(14.5 \%)$ & $89(18.7 \%)$ & \\
\hline $1500-2000$ & $10(2.1 \%)$ & $11(23 \%)$ & $22(4.6 \%)$ & $219(46.1 \%)$ & $262(55.1 \%)$ & \\
\hline$>2000$ & $1(0.2 \%)$ & $2(0.4 \%)$ & $1(0.2 \%)$ & $15(3.1 \%)$ & $19(4 \%)$ & \\
\hline Total & $22(4.6 \%)$ & $34(7.1 \%)$ & $44(9.2 \%)$ & $375(78.9 \%)$ & $475(100 \%)$ & \\
\hline
\end{tabular}

Table 3: Correlations between levels of second sampling T4 and demographic characteristics of patients.

\begin{tabular}{|c|c|c|c|c|c|c|}
\hline & $<3 \mathbf{~ N ~ ( \% ) ~}$ & 5-Mar N (\%) & 7-May N (\%) & > 7 N (\%) & Total N (\%) & P-value \\
\hline Sex & & & & & & \\
\hline Male & $3(1.4 \%)$ & $7(3.4 \%)$ & $17(8.3 \%)$ & $66(32.5 \%)$ & $93(45.8 \%)$ & 0.018 \\
\hline
\end{tabular}




\section{Academic Journal of Pediatrics \& Neonatology}

\begin{tabular}{|c|c|c|c|c|c|c|}
\hline Female & $8(3.9 \%)$ & $5(2.4 \%)$ & $10(4.9 \%)$ & $87(38.4 \%)$ & $110(54.1 \%)$ & \\
\hline Total & $11(5.4 \%)$ & $12(5.9 \%)$ & $27(13.3 \%)$ & 153(75.3\%) & $203(100 \%)$ & \\
\hline \multicolumn{7}{|l|}{ G.A (weeks) } \\
\hline $23-25$ & $1(0.4 \%)$ & $0(0 \%)$ & $0(0 \%)$ & $3(1.4 \%)$ & $4(1.9 \%)$ & 0.06 \\
\hline $25-28$ & $1(0.4 \%)$ & $2(0.9 \%)$ & $8(3.9 \%)$ & $20(9.8 \%)$ & $31(15.27 \%)$ & \\
\hline $28-32$ & $6(2.9 \%)$ & $5(2.4 \%)$ & $12(5.9 \%)$ & $82(40.3 \%)$ & $105(51.7 \%)$ & \\
\hline $32-34$ & $3(1.4 \%)$ & $3(1.4 \%)$ & $3(1.4 \%)$ & $30(14.7 \%)$ & $39(19.2 \%)$ & \\
\hline$>34$ & $0(0 \%)$ & $2(0.9 \%)$ & $4(1.9 \%)$ & $18(8.8 \%)$ & $24(11.8 \%)$ & \\
\hline Total & $11(5.4 \%)$ & $12(5.9 \%)$ & $27(13.3 \%)$ & 153(75.3\%) & $203(100 \%)$ & \\
\hline \multicolumn{7}{|c|}{ Birth Weight(grams) } \\
\hline$<750$ & $0(0 \%)$ & $1(0.4 \%)$ & $0(0 \%)$ & $3(1.4 \%)$ & $4(1.9 \%)$ & 0.036 \\
\hline $751-1000$ & $1(0.4 \%)$ & $0(0 \%)$ & $9(4.4 \%)$ & $10(4.9 \%)$ & $20(9.8 \%)$ & \\
\hline $1001-1250$ & $2(0.9 \%)$ & $3(1.4 \%)$ & $3(1.4 \%)$ & $28(13.7 \%)$ & $36(17.7 \%)$ & \\
\hline $1251-1500$ & $2(0.9 \%)$ & $3(1.4 \%)$ & $3(1.4 \%)$ & $34(16.7 \%)$ & $42(20.6 \%)$ & \\
\hline $1500-2000$ & $4(1.9 \%)$ & $5(2.4 \%)$ & $9(4.4 \%)$ & $69(33.9 \%)$ & $87(42.8 \%)$ & \\
\hline$>2000$ & $2(0.9 \%)$ & $0(0 \%)$ & $3(1.4 \%)$ & $9(4.4 \%)$ & $14(6.8 \%)$ & \\
\hline Total & $11(5.4 \%)$ & $12(5.9 \%)$ & $27(13.3 \%)$ & $153(75.3 \%)$ & $203(100 \%)$ & \\
\hline
\end{tabular}

As we shown in (Table 1), in overall in the first blood sampling, 100 of $475(21 \%)$ preterm infants had T4, lower than $7 \mathrm{ng} / \mathrm{dl}$ and 56 cases $(11.7 \%)$ had moderate to severe deficiency of T4 (lower than $5 \mathrm{ng} / \mathrm{dl}$ ) but in second sampling, in the 50 from 203 cases $(24.7 \%)$, the low levels of thyroxin hormone were observed which in 23 cases $(11.35 \%)$, the more severe deficiency was detected.

In comparisons the values of $\mathrm{T} 4$ in both of the first and second sampling: in 124 from 203 neonates (61\%) both results were normal, in 24 cases (11.8\%) persistent hypothyroxinemia was observed (both tests were low), 26(12.8\%) had delayed hypothyroxinemia (the first one was normal and the second one was lower than normal) and in 29(14.2\%) transient form of Table 4: Final results of thyroid function test of patients. hypothyroxinemia (first sampling low and the second one was in normal ranges) were detected.

In the 125 (26.9\%) cases in the first sampling and 63 (31\%) in the second one increased level of TSH was concurrent with normal levels of T4 (means the possible hypothyroidism).

Regard to the probable correlation between intraventricular hemorrhage (IVH) and HOP, we analyzed the rate of IVH in our population and we found that in 323 neonates whose brain sonography was performed ,264 cases(81.6\%) had normal brain sonography,28(8.6\%) patients had IVH grade 1,2 and $30(9.3 \%)$ neonates had IVH grade 3, 4 (Table 4).

\begin{tabular}{|c|c|c|}
\hline & First sampling N (\%) (Total=463) & Second sampling N(\%) (Total=203) \\
\hline Euthyroid & & \\
\hline$(T 4: N L(>7)$ & $238(51.4 \%)$ & $90(44.3 \%)$ \\
\hline TSH:NL(<5)) & & \\
\hline Hypothyroxinemia & & $29(14.2 \%)$ \\
\hline$(T 4 \downarrow(<7)$ & $61(13.1 \%)$ & \\
\hline TSH:NL $(<5))$ & & $14(6.8 \%)$ \\
\hline Hypothyroid & & $6(2.9 \%)$ \\
\hline$(T 4 \downarrow(<7)+T S H \uparrow(>5-10)$ & $25(5.3 \%)$ & $1(0.49 \%)$ \\
\hline TSH TSH $\uparrow(>10-20)$ & $7(1.5 \%)$ & $21(10.3 \%)$ \\
\hline TSH $\uparrow(>20)$ & $7(1.5 \%)$ & \\
\hline Total & $39(8.3 \%)$ & \\
\hline Possible Hypothyroid & & \\
\hline$(T 4: N L(>7)$ & $125(26.9 \%)$ & $63(31 \%)$ \\
\hline TSH: $\uparrow L(>5))$ & & \\
\hline
\end{tabular}




\section{Discussion}

The current study was done to detect the rate of hypothyroxinemia in our premature infants. Given that in the screening program of congenital hypothyroidism, we don't measure T4, there is concern that the cases with severe hypothyroxinemia are missed. Although in recent researches didn't confirmed the treatment of all HOP [11] as regards, very low level of T4 can cause later consequences in preterm infants and on the other hand may be the first laboratory signs of congenital permanent hypothyroidism, detecting hypothyroxinemia particularly the severe deficiency of T4 in the first days of life, emphasize the follow up thyroid function tests even after neonatal periods. In current research, we found that $22 \%$ of all cases both in the first and second samplings, $21 \%$ of 476 neonates in the first thyroid test and $24.7 \%$ of 203 patients in the second one had a low level of T4 and $11.8 \%$ in the first sampling and $11.3 \%$ in the second ones, had moderately decreased level of this hormone (lower than $5 \mathrm{ng} / \mathrm{dl}$ ). These values are the summation of all decreased levels of T4 in both of hypothyroidism and hypothyroxinemia. The rate of true hypothyroxinemia of prematurity (HOP) (T4 4 concurrent with normal TSH) in our research were $61(13.1 \%)$ out of 476 and $29(14.2 \%)$ from 203 cases.in the first and second sampling. Thyroid function status in preterm infants is different with term neonates, Immaturity of the hypothalamic-pituitary-thyroid axis can cause some abnormalities in laboratory finding that they recover overtime with increase the age of patients and maturity of the Brain-thyroid axis [12] .There are some researches that showed the too low level of thyroxin hormone can cause neurodevelopment poor outcome and they recommended the use of levothyroxine in severe deficiency of thyroid hormone in extremely low birth weight preterm neonates(ELBW) with gestational age lower than 27-28 weeks, Such as study was done by Nomura s that suggested the low dose of levothyroxine $(5-10 \mu \mathrm{g} / \mathrm{dl})$ result in better neurodevelopmental outcome in one years old of ELBW infants with low levels of T4 or clinical symptoms of hypothyroidism in neonatal periods [13]. But some other current researches such as Iijima S showed that only low thyroxin level with high levels of TSH is indication for treatment [14].In a randomized control trial at 18 months of corrected age was done by Uchiyama in very low birth weight infants with $\mathrm{TSH}<10 \mu \mathrm{U} /$ $\mathrm{ml}$ and FT4 $<0.8 \mathrm{ng} / \mathrm{ml}$ with treatment by $5 \mu \mathrm{g} / \mathrm{ml}$ levothyroxine, there wasn't any significant effect in the growth and developmental state and visual or hearing impairments of patients and they didn't suggest treatment of this transient thyroid problem in preterm infants [15].

It is interesting to know some researches such as Shannon showed that contradictory to previous studies, the higher levels of FT4 in very premature infants was concomitant with adverse neurodevelopmental outcome in the age of 7-year and childhood [16]. With results of this study, prescription of levothyroxine in patients with mild to moderate hypothyroxinemia isn't reasonable. However the incidence of permanent congenital hypothyroidism in preterm infants is the same as term babies, transient thyroid disorders are more common in premature neonates [17].In the results of our study $39(8.3 \%)$ of patients in the first sampling and $21(10.3 \%)$ in the second ones had hypothyroidism. Considering that $7(1.5 \%)$ in first one and $1(0.49 \%)$ in the second one, the level of TSH was more than $20 \mathrm{ng} / \mathrm{dl}$, the probability of permanent hypothyroidism in our patients was low. In the research by Tfayli $\mathrm{H}$, the rate of higher TSH level in preterm babies was significant and the researchers concluded that this result could be due to the use of povidone-iodine disinfectants in very low birth weight infants [18].

As the results of our study, the sex of patients had a significant effect on the T4 levels and this disorder was more common in the male sex, contrary to the congenital hypothyroidism that is female predominant [19]. The most sensitive laboratory test for diagnosing of hypothyroidism is TSH. Concurrent low level of T4 with the high level of TSH is confirming the precise diagnosis of disease. In cases with a high level of TSH particularly in slightly increase the level of this hormone, without low levels of T4, the diagnosis of hypothyroidism is not definite and repeat the test is necessary. Such as our research with relatively high numbers of patients with this state, means125 (26.9\%) in the first sampling and63 (31\%) in the second one. In our study the rate of IVH was $8.6 \%$ for IVH grade 1, 2 and 9.3\% for IVH grade 3 and 4, although based on the multifactorial basis of the IVH in premature babies, detection of the precise causative effect of the low T4 level in this disorder is difficult.

The limitation of our research was that we didn't have the prolonged outcome of preterm babies with the very low levels of thyroxin and we just had the values of hormone in neonatal periods. Another limitation of that was the lack of availability to the results of repeated thyroid test of patients after discharge from the hospital. Information about the courses of the changes in the thyroid hormone help us to the notice the final status of this hormone in premature babies. Inaccessibility to the values of free T4 was another great limitation of our study, judging by T4 without free T4 about HOP may decrease the validity of diagnosis. The strength of our research was the relatively high sample size of patients.

\section{Conclusion}

Our research resulted that the rate of hypothyroxinemia of prematurity, particularly severe forms of this disorders is remarkable and the measurement of $\mathrm{T} 4$ in frequent sampling of preterm infants can detect them for a better follow up and prevention the probable consequences of the disease. For evaluation the effect of very low thyroxin hormones in the final outcome of patient we suggest further researches with a good methodology.

\section{Acknowledgment}

We have a special thanks to the Neonatal Health Research Center, Research Institute for Children Health, Shahid Beheshti University of Medical Sciences, Mofid children's Hospital, for the supporting of our research. 


\section{References}

1. Büyükgebiz A (2013) Newborn screening for congenital hypothyroidism. J Clin Res Pediatr Endocrinol 5(Suppl 1): 8-12.

2. Dussault JH (1999) The anecdotal history of screening for congenital hypothyroidism. J Clin Endocrinol Metab 84(12): 4332-4334.

3. Salim FA, Varma SK (2014) Congenital hypothyroidism and the importance of universal newborn screening. Indian J Pediatr 81(1): 53-57.

4. Cavarzere P, Camilot M, Popa FI, Lauriola S, Teofoli F, et al. (2016) Congenital hypothyroidism with delayed TSH elevation in low-birthweight infants: incidence, diagnosis and management. Eur J Endocrinol 175(5):395-402.

5. Mehran L, Khalili D, Yarahmadi S, Delshad H, Mehrabi Y, et al. (2018) Evaluation of the congenital hypothyroidism screening programme in Iran: a 3-year retrospective cohort study. Arch Dis Childhood Fetal Neonatal Ed: fetalneonatal-2017.

6. Kilchemmann CF, Román AN, Navarrete MR, Fuentes DB (2018) Thyroid hormone Levels in very preterm neonates and extremely preterm. Rev Chil Pediatr 89(2): 202-207.

7. Delahunty C, Falconer S, Hume R, Jackson L, Midgley P, et al (2010) Levels of neonatal thyroid hormone in preterm infants and neurodevelopmental outcome at $5 \$ 1 / 2 \$$ years: millennium cohort study. J Clin Endocrinol Metab 95(11): 4898-4908.

8. Suzumura H, Nitta A, Tsuboi Y, Watabe Y, Kuribayashi R (2011). Thyroxine for transient hypothyroxinemia and cerebral palsy in extremely preterm infants. Pediatr Int 53(4): 463-467.

9. Ghirri P, Dini F, Lunardi S, Moscuzza F, Boldrini A (2015) Hypothyroxinemia in extremely low birth weight infants. Italian journal of pediatrics.
10. Rovet J, Simic N (2008) The role of transient hypothyroxinemia of prematurity in development of visual abilities. Seminars in perinatology. pp. 431-437.

11. La Gamma EF, Korzeniewski SJ, Ballabh P, Paneth N (2016) Transient Hypothyroxinemia of Prematurity. Neoreviews 17(7): e394-e402.

12. Chaudhari M, Slaughter JL (2018) Thyroid Function in the Neonatal Intensive Care Unit. Clin Perinatol 45(1): 19-30;

13. Nomura S, Ikegami H, Wada H, Tamai H, Funato M (2014) Role of levothyroxine supplementation in extremely low birth weight infants who have transient hypothyroidism without thyroid-stimulating hormone elevation. Osaka City Med J 60(1): 29-37.

14. Iijima S (2018) Current knowledge of transient hypothyroxinemia of prematurity: to treat or not to treat? J Matern Neonatal Med: p.1-7.

15. Uchiyama A, Kushima R, Watanabe T, Kusuda S (2015) Effect of l-thyroxine supplementation on infants with transient hypothyroxinemia of prematurity at 18 months of corrected age: randomized clinical trial. J Pediatr Endocrinol Metab 28(1-2): 177-182.

16. Scratch SE, Hunt RW, Thompson DK, Ahmadzai ZM, Doyle LW et al. (2014) Free thyroxine levels after very preterm birth and neurodevelopmental outcomes at age 7 years. Pediatrics 133(4).

17. Chung HR, Shin CH, Yang SW, Choi CW, Kim B Il, et al. (2009) High incidence of thyroid dysfunction in preterm infants. J Korean Med Sci 24(4): 627-631.

18. Tfayli H, Charafeddine L, Tamim H, Saade J, Daher RT, et al. (2018) Higher Incidence Rates of Hypothyroidism and Late TSH Rise in Preterm Very-Low-Birth-Weight Infants at a Tertiary Care Center. Horm Res Paediatr 89(4): 224-232.

19. Snyder PJ, Utiger RD (1972) Response to thyrotropin releasing hormone (TRH) in normal man. J Clin Endocrinol Metab 34(2): 380385.

\section{Your next submission with Juniper Publishers will reach you the below assets}

- Quality Editorial service

- Swift Peer Review

- Reprints availability

- E-prints Service

- Manuscript Podcast for convenient understanding

- Global attainment for your research

- Manuscript accessibility in different formats

( Pdf, E-pub, Full Text, Audio)

- Unceasing customer service

\section{Track the below URL for one-step submission}

https://juniperpublishers.com/online-submission.php 